\title{
Inflammation Induced Sensory Nerve Growth and Pain Hypersensitivity Requires the N-Type Calcium Channel Cav2.2
}

\author{
Saumitra Pitake1,2, Leah J. Middleton ${ }^{2}$, Ishmail Abdus-Saboor ${ }^{2}$ and \\ Santosh K. Mishra ${ }^{1,3,4,5 *}$
}

${ }^{1}$ Department of Molecular Biomedical Sciences, College of Veterinary Medicine, North Carolina State University, Raleigh, NC, United States, ${ }^{2}$ Department of Biology, University of Pennsylvania, Philadelphia, PA, United States, ${ }^{3}$ Comparative Medicine Institute, College of Veterinary Medicine, North Carolina State University, Raleigh, NC, United States, ${ }^{4}$ The W.M. Keck Center for Behavioral Biology, North Carolina State University, Raleigh, NC, United States, ${ }^{5}$ Program in Genetics, North Carolina State University, Raleigh, NC, United States

\section{OPEN ACCESS}

Edited by:

Michael Costigan,

Boston Children's Hospital, Harvard Medical School, United States

Reviewed by:

Dan McGehee,

The University of Chicago,

United States

Anindya Bhattacharya, Janssen Research and Development, United States

*Correspondence: Santosh K. Mishra

skmishra@ncsu.edu

Specialty section:

This article was submitted to

Neuropharmacology,

a section of the journal

Frontiers in Neuroscience

Received: 31 May 2019 Accepted: 05 September 2019 Published: 19 September 2019

Citation:

Pitake S, Middleton LJ, Abdus-Saboor I and Mishra SK (2019) Inflammation Induced Sensory Nerve Growth and Pain Hypersensitivity

Requires the N-Type Calcium Channel

Cav2.2. Front. Neurosci. 13:1009. doi: 10.3389/fnins.2019.01009
Voltage-gated calcium channels (VGCCs) are important mediators of pain hypersensitivity during inflammatory states, but their role in sensory nerve growth remains underexplored. Here, we assess the role of the N-type calcium channel Cav2.2 in the complete Freund's adjuvant (CFA) model of inflammatory pain. We demonstrate with in situ hybridization and immunoblotting, an increase in Cav2.2 expression after hind paw CFA injection in sensory neurons that respond to thermal stimuli, but not in two different mechanosensitive neuronal populations. Further, Cav2.2 upregulation postCFA correlates with thermal but not mechanical hyperalgesia in behaving mice, and this hypersensitivity is blocked with a specific Cav2.2 inhibitor. Voltage clamp recordings reveal a significant increase in Cav2.2 currents post-CFA, while current clamp analyses demonstrate a significant increase in action potential frequency. Moreover, CFA-induced sensory nerve growth, which involves the extracellular signal-related kinase (ERK1/2) signaling pathway and likely contributes to inflammation-induced hyperalgesia, was blocked with the Cav2.2 inhibitor. Together, this work uncovers a role for Cav2.2 during inflammation, demonstrating that VGCC activity can promote thermal hyperalgesia through both changes in firing rates of sensory neurons as well as promotion of new neurite outgrowth.

Keywords: Cav2.2, inflammation, pain, nerve growth, hyperalgesia

\section{INTRODUCTION}

Inflammation-induced pain hypersensitivity is a manifestation of increased sensory input, altered neurotransmitter release in the spinal cord, heightened excitability of neurons, and phenotypic changes brought about by afferent neurons innervating the site of inflammation (Woolf et al., 1997; Lankford et al., 1998). Release of cytokines and other inflammatory agents including bradykinin, prostaglandin E2, ATP, and a host of other agents at the site of inflammation collectively contributes toward lowering the excitability threshold of primary afferents near the affected area in the periphery (Gold and Flake, 2005; Miller et al., 2009; Fehrenbacher et al., 2012). A myriad of ionic mechanisms contribute to the increased sensory transduction observed during inflammation; these implicate changes in gene expression and voltage dependence of voltage-gated $\mathrm{Na}^{+}, \mathrm{K}^{+}$, and $\mathrm{Ca}^{2+}$ 
channels and ligand-gated ion channels such as transient receptor potential (TRP channels) and purinergic receptors (Xu and Huang, 2002; Amaya et al., 2006; Yu et al., 2008; Lu et al., 2010).

Several studies have demonstrated that voltage-gated calcium channels (VGCCs) play an important role in inflammationinduced hyperexcitability of primary afferents (Schaible et al., 2000; Zhang and Dougherty, 2014; Berta et al., 2017). Although almost all subtypes of VGCCs (N, P/Q, R, and T) are expressed throughout the central nervous system (Hillman et al., 1991; Westenbroek et al., 1992; Zamponi et al., 2015). Recent studies have highlighted the role that N-type (Cav2.2) channels play in neuropathic and inflammatory pain (Snutch, 2005; Altier et al., 2007; Lee, 2013). Cav2.2 channel is expressed abundantly in dorsal root ganglion (DRG) neurons and genetic and pharmacological strategies that block Cav2.2 activity are antinociceptive (Woolf et al., 1997; Lankford et al., 1998; Gold and Flake, 2005; Miller et al., 2009; Fehrenbacher et al., 2012). Importantly, intracellular calcium signaling has been shown to regulate neurite growth patterns in neurons through VGCC channels (Vigers and Pfenninger, 1991; Weiss, 2008). In PC12 neural cell line, calcium influx through VGCC causes extensive neurite outgrowth (Solem et al., 1995). In another study in parasympathetic cultured neurons, it has been shown that blockade of calcium influx through L-type and N-type VGCCs as well as transient receptor potential canonical (TRPC) channels, reduces the growth of neurite processes while release from intracellular stores was not significantly affected (Zamburlin et al., 2013). Additionally, Xenopus live imaging findings, coupled with studies of cultured Xenopus spinal neurons, demonstrate that an optimal frequency of calcium transients regulates neurite extension (Gu and Spitzer, 1995). However, it remains unclear whether this same phenomenon drives the afferent sensory growth in inflammation, which leads to pain hypersensitivity at the periphery, and furthermore which downstream signaling cascades are involved in sensory afferent outgrowth.

In this study, we investigated the role of Cav2.2 in sensory nerve outgrowth during an inflammatory pain state. By employing in vitro and in vivo cellular, pharmacological, behavioral, and physiological methods, we revealed a function for the Cav2.2 channel as a modulator of sensory nerve growth during inflammation-induced hyperalgesia. Selective blockade of Cav2.2 channels leads to a decrease in neurite outgrowth and an attenuation of thermal, but not mechanical pain. We demonstrate with RNAscope technology that thermal hyperalgesia during inflammation is likely mediated by Cav2.2 upregulation in TrpM8+,TrpV1+, and TrpA1+ sensory neurons. Based on these findings we provide a mechanism for CFA-induced inflammatory pain hypersensitivity; wherein Cav2.2 is the primary effector and ERK1/2-MAPK is the downstream target.

\section{MATERIALS AND METHODS}

\section{Animals}

Six to eight-weeks old C57BL/6 mice of either sex were used for all the experiments and mice were purchased from the Jackson Laboratory. All procedures were conducted according to animal protocols approved by the university Institutional Animal Care and Use Committee (IACUC) at North Carolina State University and the University of Pennsylvania in accordance with National Institutes of Health (NIH) guidelines. All mice were group housed with four/five animals per cage.

\section{Drugs}

$\omega$-conotoxin GVIA (C9915) was purchased from Sigma-Aldrich and dissolved in phosphate buffered saline (PBS; $\mathrm{pH}$ 7.4). Nifedipine (481981) was purchased from Calbiochem and was dissolved in DMSO.

\section{Behavioral Assays Thermal}

A Hargreaves' test apparatus (Ugo Basile) was used to measure thermal responses to radiant heat. Mice were placed in individual chambers for $10 \mathrm{~min}$ for habituation. A radiant heat source was focused on the paws and withdrawal latency was recorded (Mishra et al., 2011). Dry ice was used to assess the acute temperature sensitivity (Brenner et al., 2012). Each animal was tested at least twice to address the variability in responses.

\section{Mechanical}

Mechanical test was performed using a von-Frey apparatus (Ugo Basile). An electronic von-Frey filament was used to measure force in grams applied to the paw and the cutoff force was set at $50 \mathrm{~g}$. Mice were habituated in the plexiglass chambers for 10 min prior to the experiment. Minimum of four measurements were taken from each paw, and the average force at which paw withdrawal was observed and reported accordingly (Mishra et al., 2011).

\section{CFA-Induced Inflammation}

Sterile complete Freund's adjuvant (CFA; $20 \mu \mathrm{l})$ was injected into the plantar surface of the hind paw to induce inflammation. The site of injection and the surrounding area was observed $24 \mathrm{~h}$ post injection to visually confirm inflammation. Mice were then tested for thermal, cold, and mechanical pain sensitivity. As a control, PBS $(20 \mu \mathrm{l})$ was injected into the plantar surface, and mice were then subjected to the same behavioral testing. The observer was blinded during all behavior recordings.

\section{Intrathecal Injection}

Mice were anesthetized briefly under isoflurane. Cav2.2 inhibitor conotoxin $(300 \mathrm{pmol} / 10 \mu \mathrm{l})$ and vehicle PBS $(10 \mu \mathrm{l})$ were injected in between the L5 and L6 lumbar space using a 30-gauge insulin syringe, and a tail flick indicated successful entry of the needle into the subdural space. The injection was repeated every $24 \mathrm{~h}$ for 3 days. Baseline behavior was recorded immediately following intrathecal injection of vehicle and/or conotoxin and subsequent analyses were performed 24 and $72 \mathrm{~h}$ post-injection to assess the results of the specific treatments. All the behavior analyses were performed by an observer who was blinded to the treatment groups. 


\section{Western Blot}

To extract total protein, dorsal root ganglia, paws, and sciatic nerves were homogenized using a tissue homogenizer in the presence of $100 \mu \mathrm{l}$ of ice cold RIPA buffer supplemented with protease inhibitor tablets $l\left(\right.$ Pierce $\left.^{\mathrm{TM}}\right)$. Total protein of lysates was measured using standard BCA (Bicinchoninic Acid Assay). Protein lysates were then denatured by heating at $95^{\circ} \mathrm{C}$ in Laemmli's buffer containing $2 \% \mathrm{w} / \mathrm{v}$ SDS, $62.5 \mathrm{mM}$ Tris ( $\mathrm{pH}$ 6.8), $10 \%$ glycerol, $50^{\circ} \mathrm{mM} \mathrm{DTT}$, and $0.01 \% \mathrm{w} / \mathrm{v}$ bromophenol blue. The lysates were cooled on ice and briefly micro-centrifuged. Aliquots of $35^{\circ} \mu \mathrm{g}$ of protein were loaded onto a $4-12 \%$ SDS-PAGE gel, and subsequently electro blotted onto PVDF membranes. Membranes were incubated in $15 \mathrm{ml}$ of blocking buffer [ $20 \mathrm{mM}$ Tris base and $140 \mathrm{mM} \mathrm{NaCl}, 5 \%$ bovine serum albumin (BSA), and $0.1 \%$ Tween-20] for $1 \mathrm{~h}$. Membranes were then incubated with the desired primary antibody diluted in $10 \mathrm{ml}$ of blocking buffer at $4^{\circ} \mathrm{C}$ overnight. Next day membrane was washed and incubated with an appropriate horseradish peroxidase-conjugated secondary antibody (1:1000) to detect proteins in $10^{\circ} \mathrm{ml}$ blocking buffer for $1 \mathrm{~h}$ at room temperature. Immuno-reactive proteins were revealed using enhanced chemiluminescence detection (Pierce ECL ${ }^{\mathrm{TM}}$ ). AntiTUJ1 (Cat. MMS-435P) was purchased from Covance, AntiCav2.2 (Cat. sc-271010), Anti-GAPDH (Cat. sc-32233) were purchased from Santa Cruz Biotechnology. Anti-ERK 1/2 (Cat. 4695) and Anti-phospho-ERK 1/2 (Cat. 4370) were purchased from Cell Signaling technology. All primary antibodies were used at a dilution of 1:100 except for Anti-Cav2.2 which was used at a dilution of 1:100. Secondary anti-rabbit and anti-mouse antibodies were purchased from Santa Cruz Biotechnology. Alexa 488 and Alexa 555 conjugated secondary antibodies were purchased from Thermo Fisher Scientific. The intensity of each protein band was analyzed using an opensource software Image $(\mathrm{NIH})$. The expression of protein was normalized to the expression of loading control GAPDH. For phosphorylated ERK 1/2 expression, Total extracellular signal-related kinase (ERK) was used as a control.

\section{Immunohistochemistry/ Immunocytochemistry}

Sciatic nerve were dissected from mice and quickly frozen in Tissue-Tek ${ }^{\circledR}$ O.C.T. compound over dry ice. $10-12^{\circ} \mu \mathrm{M}$ sections were cut using cryostat and were placed on charged slides. The sections were then fixed using 4\% Paraformaldehyde solution for $20 \mathrm{~min}$ and then quickly rehydrated with PBS for $5 \mathrm{~min}$. Sections were then incubated in a $5 \%$ BSA solution containing $0.1 \%$ Triton for better antigen exposure for $45 \mathrm{~min}$. The sections were then washed with PBS and incubated with 5\% BSA solution with desired primary antibodies at $4^{\circ} \mathrm{C}$ overnight. Alexa conjugated secondary antibodies were used along with DAPI stain containing mounting medium and sections were imaged using Leica DM5000b microscope. For immunocytochemistry, mice were unilaterally injected in the hind paw with CFA, and ipsilateral and contralateral lumbar DRGs were harvested $24 \mathrm{~h}$ later. To assess the effect of conotoxin on neurite growth and Cav2.2 expression, ipsilateral DRGs from control (vehicle treated), CFA and CFA + conotoxin treated mice were harvested after $24 \mathrm{~h}$. DRGs were then dissociated into single cells and plated at equal density on glass coverslips. The cells were fixed with $4 \%$ paraformaldehyde for $15 \mathrm{~min}$ and then quickly rehydrated with PBS for 5 min. Immunostaining was performed as described above using antibodies against TUJ1 (1:1000) and Cav2.2 (1:1000).

\section{RNAScope in situ Hybridization}

C57BL/6 mice were injected with CFA $(20 \mu \mathrm{l})$ unilaterally in the right hind paw and ipsilateral and contralateral lumbar dorsal root ganglia (L4-L5) were harvested 48 h post treatment. Dorsal root ganglia were quickly frozen over dry ice in Tissue-Tek ${ }^{\circledR}$ O.C.T. 10-12 $\mu \mathrm{M}$ sections were cut using a cryostat (Leica CM3050S) and were placed on charged slides. The sections were further processed according to RNAscope ${ }^{\circledR}$ Multiplex Fluorescent Assay v2 (Advanced Cell Diagnostics, Hayward, CA, United States) protocol using target probes for Cav2.2, TRPV1, TRPM8, TRPA1, MrgprD, and MrgprB4. RNAScope results were examined using Leica DM6000FS confocal microscope at $20 \times$ magnification. Fifteen cells with detectable signal were selected at random for quantification by a blinded observer who was unaware of the treatment groups. The signal intensity of mRNA clusters observed within each cell was analyzed by drawing a region of interest (ROI) around each cell and mean signal intensity in arbitrary units generated by ImageJ software was noted. The dimensions of the ROI were kept constant throughout the analysis to avoid bias. This process was repeated for each channel (red, green, and blue) including overlay images. At least 15 ROIs were drawn per section and at least five ipsilateral and five contralateral sections were analyzed from $n=3$ mice. The entire quantification was performed by an observer in a manner blinded to mRNA probes and channel assignments.

\section{Electrophysiology Spontaneous Action Potential Recording in Dissociated DRG Neurons}

Whole cell current clamp recording was carried out in dissociated DRG neurons after plating them overnight. Thin walled borosilicate glass capillaries (TW150-4) were purchased from World Precision Instruments and electrodes were pulled using Sutter P-97 puller. The pipette resistance was between 2 and $3 \mathrm{M} \Omega$ in bath solution. For current-clamp recording, the internal solution contained $145 \mathrm{mM} \mathrm{K}$-gluconate, $2 \mathrm{mM}$ $\mathrm{MgCl}_{2}, 1 \mathrm{mM} \mathrm{CaCl}_{2}, 5 \mathrm{mM} \mathrm{K}$ GTP, $5 \mathrm{mM}$ HEPES, and $10 \mathrm{mM}$ EGTA adjusted to $\mathrm{pH} 7.4$ with $\mathrm{KOH}$ and the bath solution contained $140 \mathrm{mM} \mathrm{NaCl}, 5 \mathrm{mM} \mathrm{KCl}, 2 \mathrm{mM} \mathrm{CaCl}_{2}, 1 \mathrm{mM}$ $\mathrm{MgCl}_{2}, 10 \mathrm{mM}$ HEPES, and $10 \mathrm{mM}$ Glucose adjusted to $\mathrm{pH}$ 7.4 with $\mathrm{NaOH}$. Electrophysiologic recording was initiated in voltage clamp mode until attaining a stable whole-cell clamp and then switched to current clamp mode. DRG neurons were held at $0 \mathrm{pA}$, and the spontaneous action potentials were recorded in "zero current clamp" mode. Number of spontaneous action potentials observed in a fixed time interval were used to assess hypersensitivity between different treatment groups. Spontaneous action potentials were recorded from small 
diameter $(<45 \mu \mathrm{M})$ DRGs dissociated from the lumbar region. The diameter was determined using $20 \times$ Nikon objective and scale generated by Nikon Elements software. Only neurons with a resting membrane potential of at least $-40 \mathrm{mV}$ and stable baselines were used for further experiments and analysis. Recordings were included only if the above-mentioned criteria were met. This was done to minimize spontaneous firing caused by deterioration or other artifacts during recording.

\section{Cav2.2 Current Recordings}

Internal solution for voltage clamp recordings contained (in $\mathrm{mM}$ ): $140 \mathrm{Cs}$-Aspartate, $10 \mathrm{Cs} 2 \mathrm{EGTA}, 5 \mathrm{MgCl}_{2}$, and $10 \mathrm{HEPES}$, $\mathrm{pH}$ 7.4. To isolate $\mathrm{N}$-type $\mathrm{Ca}_{2}+$ currents, the $\mathrm{K}+$ currents and L-type $\mathrm{Ca}^{2+}$ currents were blocked with tetraethylammonium-Cl (TEA-Cl) and $1 \mu \mathrm{M}$ nifedipine (Calbiochem), respectively. The standard external solution was composed of (in mM): 137.5 TEA$\mathrm{Cl}, 10 \mathrm{CaCl}_{2}, 10 \mathrm{HEPES}, \mathrm{pH}$ 7.4. Electronic compensation was used to reduce the effective series resistance $(<5 \mathrm{M} \Omega)$. Currents were filtered at $2.9 \mathrm{KHz}$ using a 4-pole Bessel filter. In most of the recordings, a $1 \mathrm{~s}$ prepulse to $-20 \mathrm{mV}$ followed by a $50 \mathrm{~ms}$ repolarization to $-50 \mathrm{mV}$ was administered before the test pulse to inactivate T-type calcium channels. I-V relationships were generated by applying 13 pulses from a holding potential of $-70 \mathrm{mV}$ in $10 \mathrm{mV}$ increments. Pulse duration was fixed at $250 \mathrm{~ms}$. Cav2.2 currents were recorded at their peak activation, at $+10 \mathrm{mV}$, for all groups. Cell capacitance $(\mathrm{pF})$ was used to normalize currents observed $(\mathrm{pA})$ at $+10 \mathrm{mV}$ for all groups and the resulting current density $(\mathrm{pA} / \mathrm{pF})$ was used for statistical comparisons. All recordings were made at room temperature. All analyses were carried out using Clampfit software and only small-medium diameter $(<45 \mu \mathrm{M})$ DRG neurons were used for recordings.

\section{Quantification and Statistical Analysis}

Mice were randomly assigned to treatment groups. Behavior and in situ hybridization analyses were performed in a manner blinded to treatment assignments. Data are expressed as mean \pm standard error of mean (SEM) and analyzed using GraphPad Prism 7.0. When comparing two treatment groups to one another student's test was used. One-way ANOVA was applied to analyze repeated measurements from behavioral tests followed by Dunn's post hoc test for multiple comparison. Oneway ANOVA with Holm-Sidak test was applied to analyze electrophysiology and western blot data. All statistical tests were two-tailed, and the level of significance was set at $p<0.05$.

\section{RESULTS}

\section{Co-expression of Cav2.2 and Nociceptive Markers in Normal and Inflammatory Conditions}

To determine which molecular populations of nociceptors might alter their expression of Cav2.2 during CFA-induced inflammatory states, we used RNAscope technology to perform in situ hybridization for Cav2.2 and a panel of nociceptive markers. To induce inflammation, we performed unilateral intra-plantar injections of CFA, and $48 \mathrm{~h}$ post-injection we performed both two- and three-color in situ hybridization with RNAscope probes for Cav2.2 in conjunction with $\operatorname{Tr} p V 1, \operatorname{Tr} p M 8$, and $\operatorname{Trp} A 1$, which are markers of thermal (hot and cold) and noxious stimuli, respectively (Figures 1A-D). Comparing the ipsilateral CFA-injected side to the control contralateral side, we observed a statistically significant increase in Cav2.2 expression in $\operatorname{Tr} p V 1+, \operatorname{Tr} p M 8+$, and $\operatorname{Tr} p A 1+$ nociceptors (Figures 1G-I). Conversely, such an increase was not observed when we performed two-color in situ hybridization with RNAscope probes for Cav2.2 and Mrgprd, which is a marker for mechanosensitive non-peptidergic nociceptors (Figures 1E,F,I). Consistent with this finding, we did not observe significant co-labeling of Cav2.2 with Mrgprb4 C-fiber low-threshold mechanoreceptors in the ipsilateral or contralateral side postCFA (Supplementary Figure S1). Taken together, these results are consistent with a role for Cav2.2 in thermal, but not mechanical hyperalgesia. The RNAscope results are consistent with increases in Cav2.2 protein expression during inflammation, and we further confirmed this by performing western blot analyses with an antibody specific to Cav2.2, and we observed a statistically significant increase in protein expression comparing the control (contralateral) versus CFA (ipsilateral) injected mice (Figures 1K,L).

\section{Conotoxin Inhibits CFA-Induced Thermal, but Not Mechanical Hyperalgesia}

Next, we sought to determine if blocking Cav2.2 action with $\omega$-conotoxin, a potent inhibitor or Cav2.2, could prevent the development of hyperalgesia in vivo. Responsiveness to heat, cold, and mechanical stimuli were recorded during basal conditions and 24 and $72 \mathrm{~h}$ following unilateral CFA injection into the hind paw (Figure 2A). Consistent with prior studies, we observed hypersensitivity to heat, cold, and mechanical stimuli in CFA-injected mice compared to control vehicle injections (Figures 2B-D). To assess the inhibitory impact of conotoxin in attenuating this hypersensitivity, mice were administered either PBS or conotoxin intrathecally every $24 \mathrm{~h}$ for 3 days prior to behavioral assays (Figure 2A). We found that conotoxin administration reduced heat hyperalgesia in the Hargreaves' thermal test at 24 and $72 \mathrm{~h}$, preventing the reduced withdrawal latency observed with CFA alone (Figure 2B). Conotoxin also reduced cold hyperalgesia in the dry ice thermal test at 24 and $72 \mathrm{~h}$, preventing the reduced withdrawal latency observed with CFA alone (Figure 2C). Next, we tested conotoxin's effects on CFA-induced mechanical hypersensitivity using von Frey hair filaments and measuring the mechanical force threshold as a behavioral readout (Figure 2D). In this mechanosensitive paradigm, unlike our results with thermal hyperalgesia, we noticed that conotoxin treatment was unable to block nociceptive hypersensitivity to mechanical stimuli at either timepoint. Together, these behavior results implicate a role for the Cav2.2 channel in thermal, but not mechanical hyperalgesia coincident with inflammatory states. 

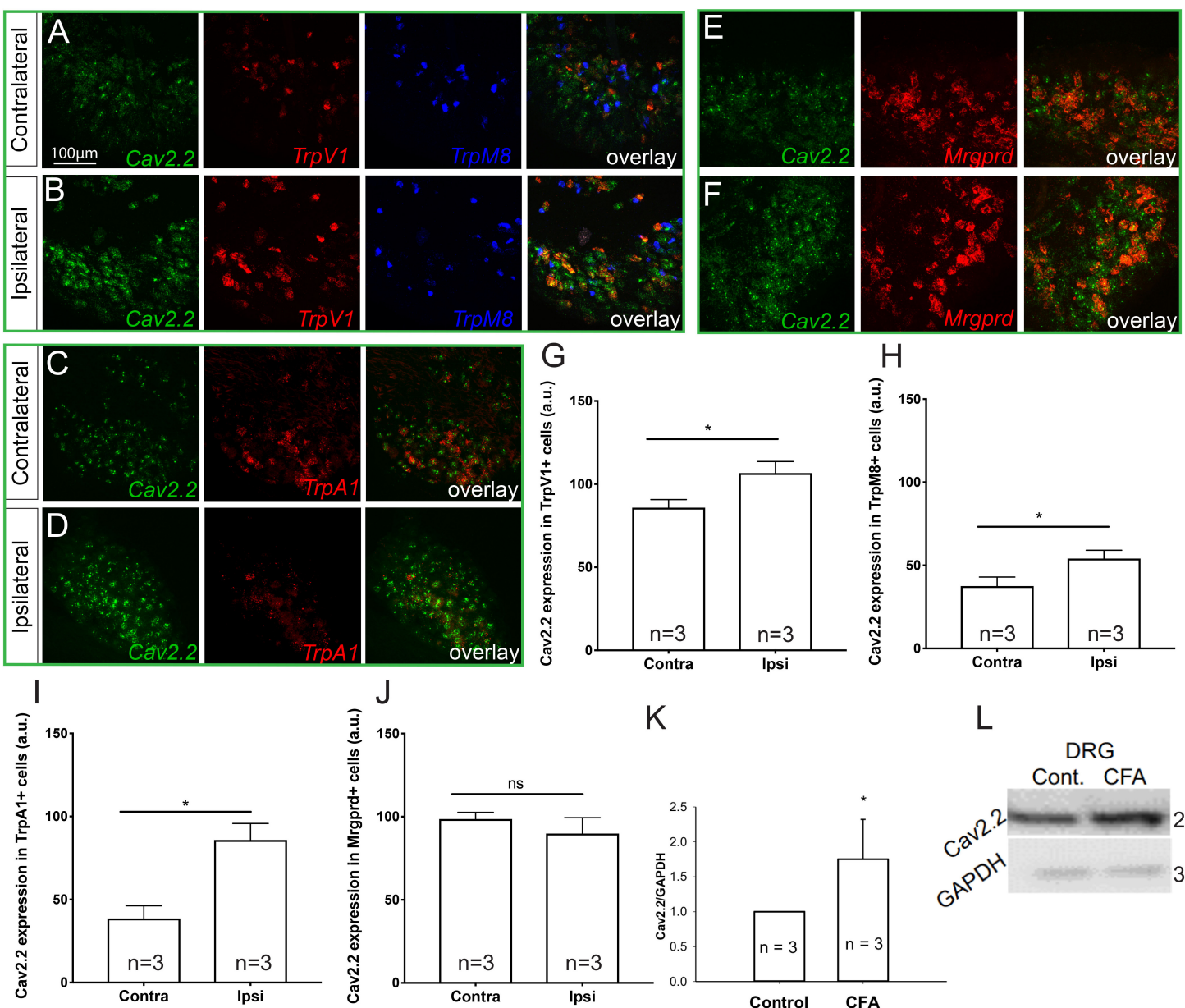

K
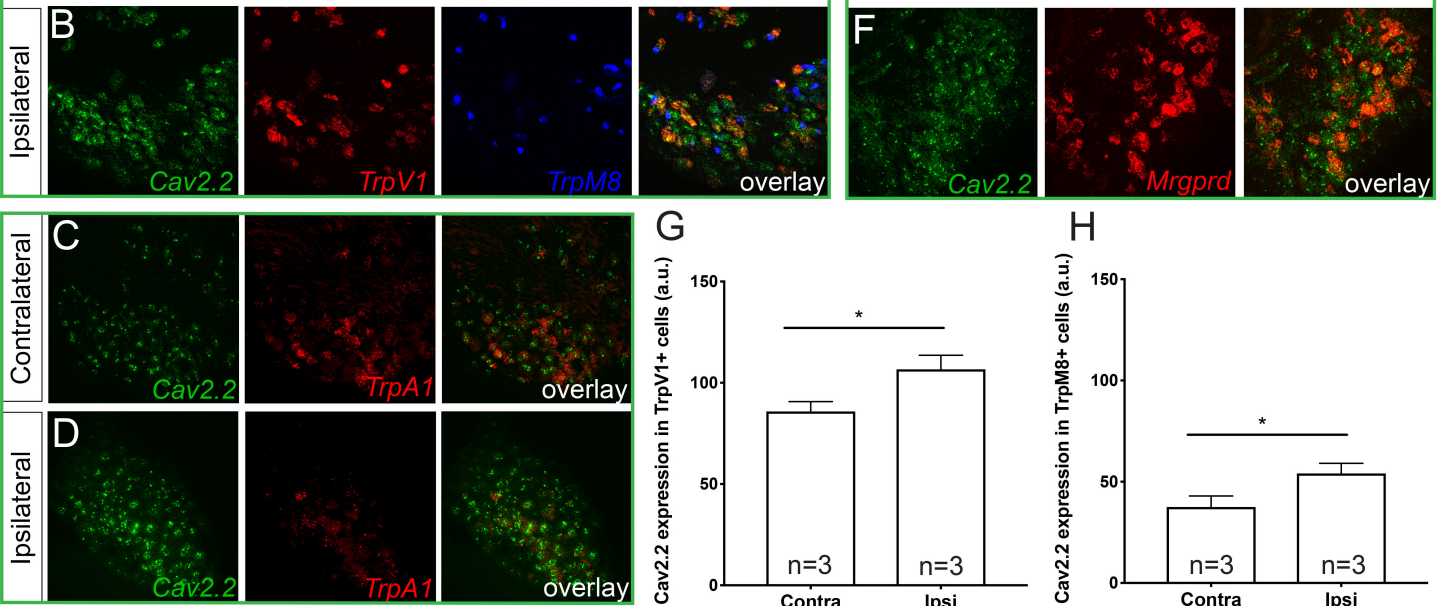

L

DRG

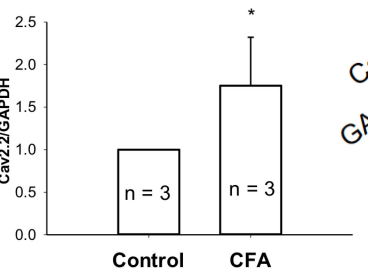

$250 \mathrm{kDa}$

$37 \mathrm{kDa}$

FIGURE 1 | Cav2.2 RNA and protein are upregulated in thermal and not mechanical sensitive nociceptors after CFA-induced inflammation. In situ hybridization with gene specific RNAscope probes of contralateral (non-treated) (A,C,E) and ipsilateral (treated) (B,D,F) DRGs showing Cav2.2 mRNA (green) in heat-sensing

TRPV1 + nociceptors (red), cold-sensing TRPM8 + nociceptors (blue), noxious-stimuli sensing TRPA1 + nociceptors (red), and mechanical-sensing

MrgprD + nociceptors (red) observed $48 \mathrm{~h}$ post CFA induced inflammation. (G-J) Mean relative fluorescence intensity of Cav2.2 mRNA (expressed in "arbitrary units" defined by NIH/ImageJ software) in TRPV1+, TRPA1+, TRPM8+, and MrgprD + nociceptors in ipsilateral and contralateral DRGs. A significant increase in Cav2.2 mRNA in neuronal populations co-expressing TRPV1, TRPA1, and TRPM8, but not MrgprD mRNA, was observed after inflammation. $N \geq 5$ sections per group obtained from three mice. ${ }^{*} P<0.05$ comparing ipsilateral to contralateral DRGs with student's $t$-test. Error bars represent standard error of the mean. (K,L) An increase in total Cav2.2 protein expression was observed in ipsilateral lumbar DRGs after CFA induced inflammation. ${ }^{*} P<0.05$ comparing ipsilateral to contralateral DRGs with student's $t$-test. Error bars represent standard error of the mean.

\section{Electrophysiological Measurement of Sensory Neurons From Normal and Inflammatory Conditions}

To complement the behavioral experiments and gain insight into the physiological properties of sensory neurons in relation to Cav2.2 during inflammatory conditions, we performed a panel of electrophysiological experiments in culture. Voltage clamp analyses revealed a significant increase in Cav2.2 current density in ex vivo conditions (Figures $\mathbf{3 A}, \mathbf{B}$ ). The specificity of these current recordings was established by using the selective blocker of Cav2.2, conotoxin (300 pmol), and this resulted in a significant reduction in current recordings (Figure 3). To assess the contribution of Cav2.2 channels to neuronal excitability, ipsilateral and contralateral lumber DRGs post CFA inflammation were isolated and spontaneous action potentials were recorded in current clamp mode (Figure 4A). Contralateral DRGs were unaffected by CFAinduced inflammation and rarely generated spontaneous action potentials while a significant increase in spontaneous action potentials was observed in ipsilateral DRGs (Figures 4B,D), and this was reduced significantly by conotoxin application (Figures 4C,D). These results demonstrate that CFA alters the intrinsic firing patterns of sensory neurons during inflammation in a Cav2.2 dependent manner.

\section{Increase in Sensory Nerve Density During Inflammation}

To examine additional alterations of sensory neurons during inflammation that may require Cav2.2, mice were unilaterally injected in the hind paw with CFA, and ipsilateral and 
A

C

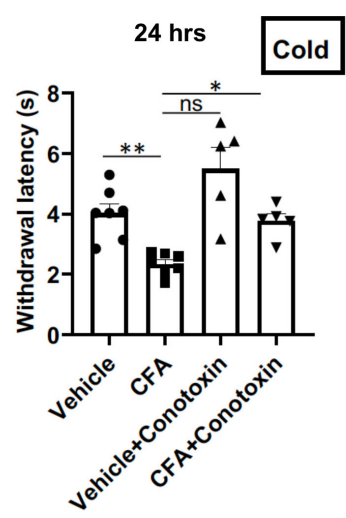

B

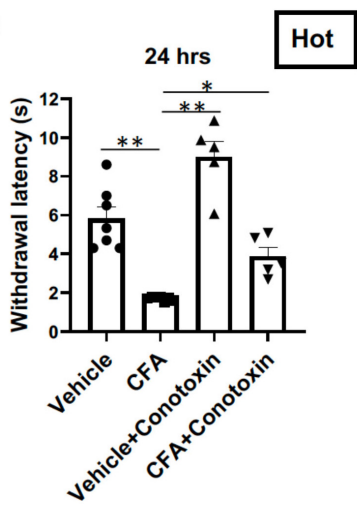

D

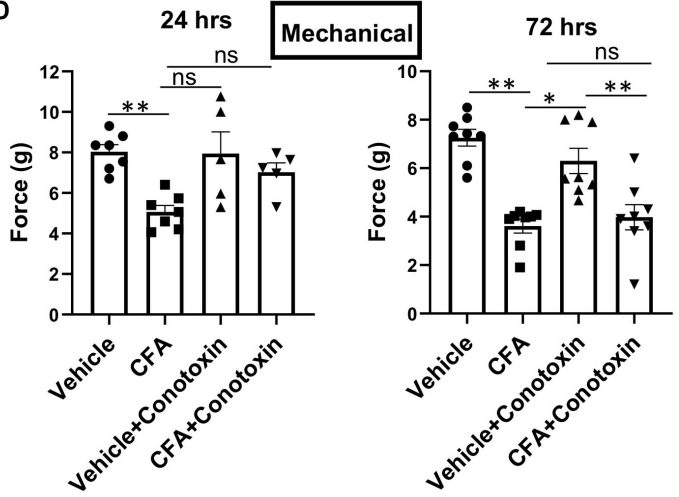

FIGURE 2 | Effects of $\omega$-conotoxin on inflammation induced pain behavior. (A) Shows a schematic representation of the behavior testing paradigm. Baseline thermal pain, acute cold pain, and mechanical pain sensitivities were recorded before CFA injection. CFA (20 $\mu$ l) was injected intra-plantar in 6-10-week-old C57Bl6 mice with intrathecal $\omega$-conotoxin $(300 \mathrm{pmol} / 10 \mu \mathrm{l})$ and/or PBS $(10 \mu \mathrm{ll}$ ). Acute thermal pain (Hargreaves'), cold pain (Dry Ice), and mechanical pain (von-Frey) were recorded 24 and $72 \mathrm{~h}$ post CFA injection. (B) Inflammation induced thermal hyperalgesia was analyzed by Hargreaves' test, (C) dry ice test, and (D) Mechanical hyperalgesia was assessed by von-Frey apparatus, $n \geq 5$ mice, ${ }^{*} P<0.05$, ${ }^{* *} P<0.01$. All statistical tests were performed as appropriate using a one-way ANOVA followed by Dunn's post hoc test for multiple comparisons within a given behavioral test and time point (either 24 or $72 \mathrm{~h}$ ).

A

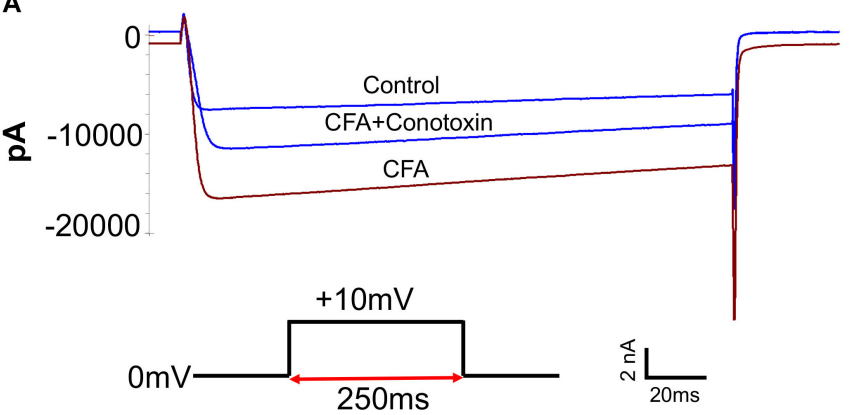

B

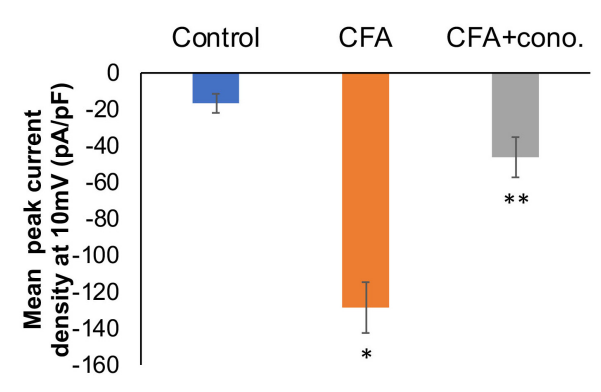

FIGURE 3 | Complete Freund's adjuvant induced inflammation leads to increased calcium currents via Cav2.2. (A) Overlay of representative raw Cav2.2 current traces from contralateral (control), ipsilateral (CFA-injected right side), and CFA + $\omega$-conotoxin treated ipsilateral DRGs at +10 mV. (B) Quantification of peak current densities at $+10 \mathrm{mV}$ for all groups. A significant increase in calcium currents can be seen upon CFA treatment which was diminished post $\omega$-conotoxin treatment, $N \geq 8$ cells per group obtained from three mice per group, ${ }^{*} P<0.05$ compare to control, ${ }^{* *} P<0.01$ compared to CFA, using one-way ANOVA followed by Holm-Sidak testing for multiple comparisons.

contralateral lumbar DRGs were harvested 24 h later. A phase contrast image of ipsilateral DRGs from CFA-injected mice revealed a significant increase in neurite formation (Figure 5A). We then used immunohistochemistry to identify sensory neurites by using the neuronal marker TUJ1, and we observed a significant increase in neuronal branching and growth only in DRG neurons ipsilateral to the CFA injection (Figure 5B). Next, we examined TUJ1 expression via western blot with protein extracted from the hind paw, DRG, and the sciatic nerve and found a significant increase in TUJ1 expression in all tissues examined (Figure 5C). 


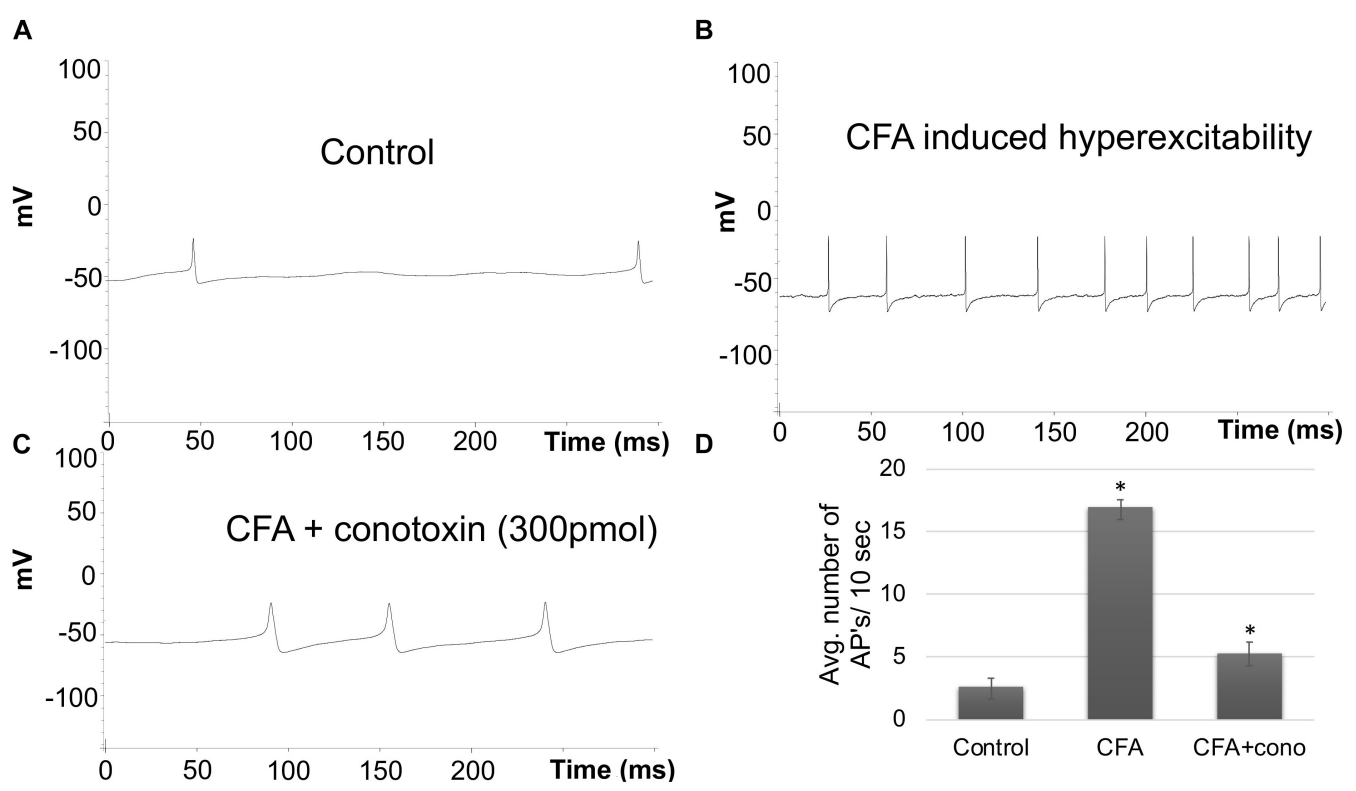

FIGURE 4 | Effects of CFA and $\omega$-conotoxin on spontaneous action potentials in DRGs. (A) Representative raw trace of spontaneous action potentials induced by changes in membrane potential in contralateral (non-treated) lumbar DRGs. (B) Representative raw trace showing an increase in spontaneous firing in ipsilateral DRGs post CFA-induced inflammation. (C) Representative raw trace showing a reduction in spontaneous firing behavior of DRGs post CFA-induced inflammation with the addition of conotoxin treatment. (D) Quantification of spikes per $10 \mathrm{~s}$ time interval, $N \geq 18$ cells per group obtained from $n=3$ mice per group, ${ }^{*} P<0.05$, using one-way ANOVA followed by Holm-Sidak testing for multiple comparisons.

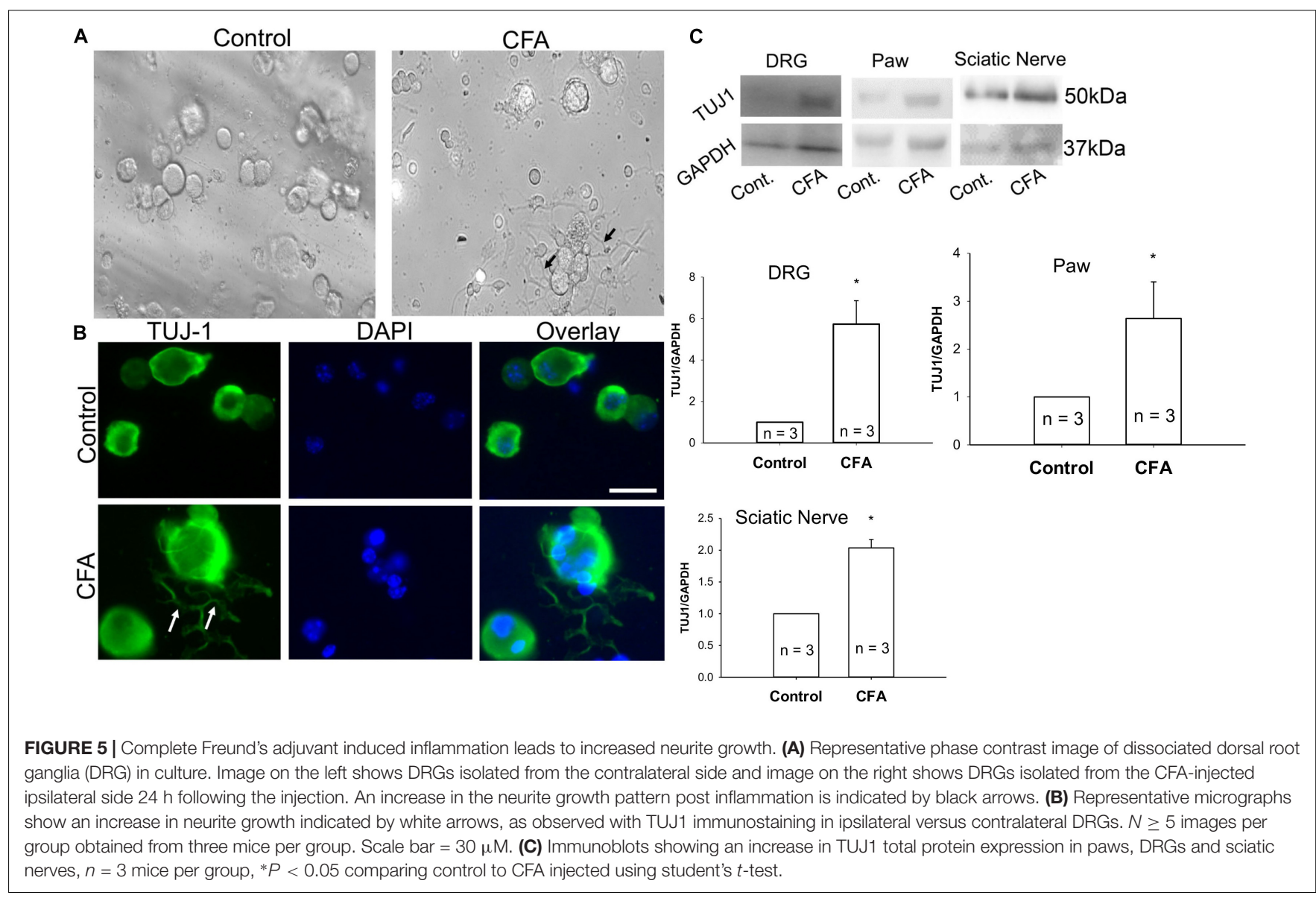


Overall, our data reveals an increase in the sensory nerve outgrowth during inflammatory conditions.

\section{Inhibiting Cav2.2 Activity Blocks Sensory Neurite Outgrowth During Inflammation}

To determine if Cav2.2 activity is necessary for the neurite outgrowth which we observed after CFA-induced inflammation, we harvested ipsilateral DRGs from mice treated with vehicle, CFA and CFA + conotoxin after $24 \mathrm{~h}$ and performed TUJ1 and Cav2.2 immunolabelling on dissociated cells (Figure 6A). Our immunostaining results using TUJ1 as a sensory neurite marker determined that Cav2.2 is required for the observed sensory neurite outgrowth phenotype (Figure 6A). Consistent with results described above in Figure 1, as well as published findings ( $\mathrm{Lu}$ et al., 2010), we observed an increase in Cav2.2 protein expression in dissociated sensory neurons following CFA paw injection (Figure 6B). We further quantified the levels of
Cav2.2 and TUJ1 proteins by western blotting in CFA and control mice, and we found an increase in TUJ1 expression, consistent with previous findings (Figure 5), upon CFA inflammation (Figures 6C-E). Interestingly, we observed a decrease in the level of TUJ1 (in the DRG and in the paw) and Cav2.2 (in the DRG) in mice $24 \mathrm{~h}$ after intrathecal administration of conotoxin (Figures 6C-F). Taken together, these data provide evidence for the requirement of Cav2.2 in CFA induced sensory neurite outgrowth.

\section{Extracellular Signal-Related Kinase Signaling Involved in Sensory Nerve Growth During Inflammation}

Several reports implicate the involvement of the ERK signaling pathway in neurite growth (Sgambato et al., 1998; Chen et al., 2011). We therefore sought to determine if this pathway might likewise be involved with Cav2.2-dependent sensory
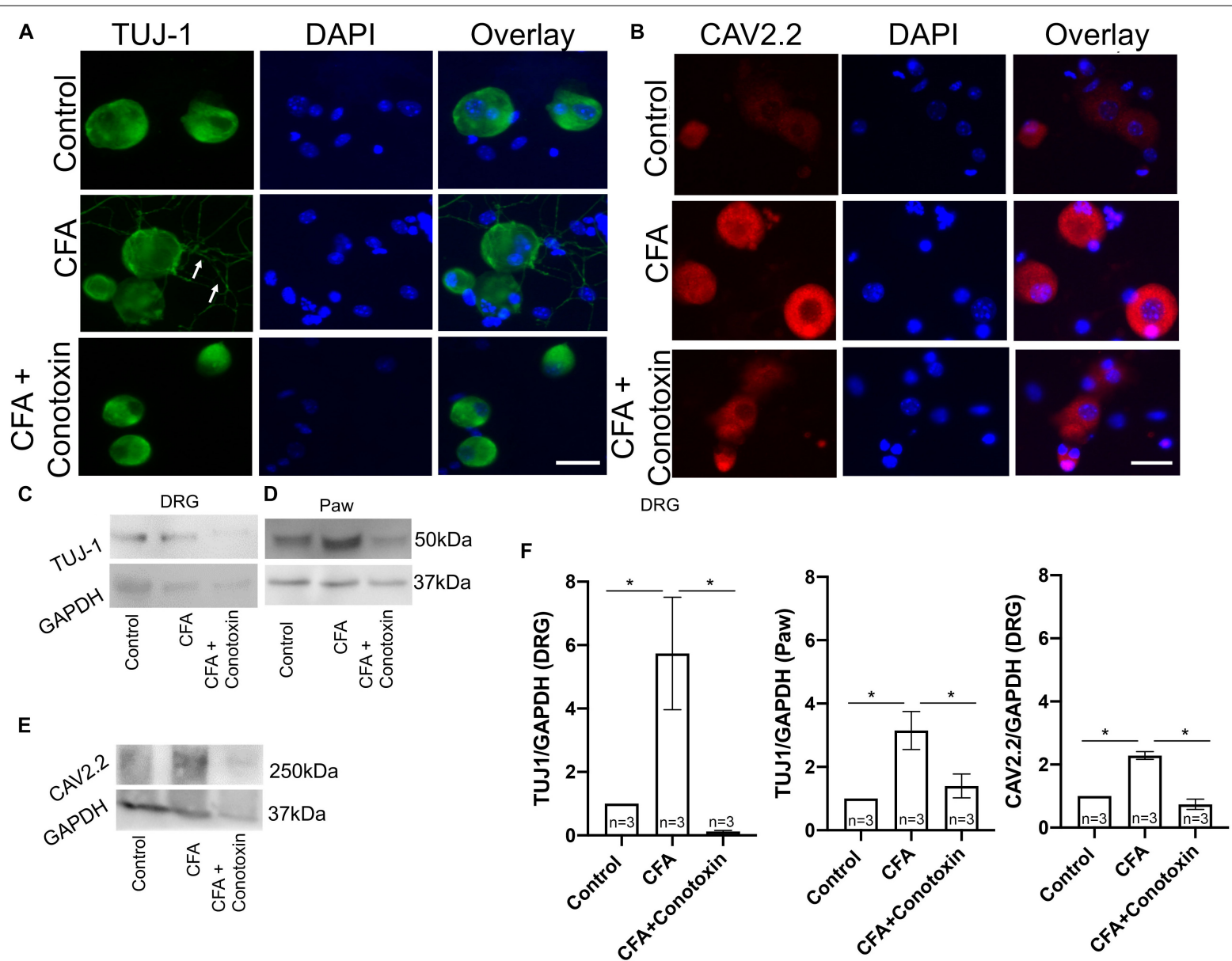

FIGURE 6 | Effects of $\omega$-conotoxin on peripheral neuronal growth and Cav2.2 protein expression. (A) Representative micrographs show TUJ1 immunolabeling in DRGs. A significant increase in TUJ1 labeled neurites can be seen post CFA inflammation (white arrows), and this is not observed post $\omega$-conotoxin treatment. (B) Representative micrographs show an increase in Cav2.2 protein expression post CFA as revealed by antisera detected against Cav2.2 CFA. This increase in Cav2.2 expression is not observed when combining CFA with $\omega$-conotoxin treatment. $N \geq 5$ images per group obtained from three mice. Scale bar $=30 \mu \mathrm{M}$. (C, $\mathbf{D})$ Immunoblots show an increase in TUJ1 protein expression in DRGs and paws. (E) Immunoblots show an increase in Cav2.2 protein expression post CFA treatment in DRGs, which is not observed combining CFA with $\omega$-conotoxin treatment. (F) Quantification of TUJ1 and Cav2.2 immunoblots, $n=3$ mice per group, ${ }^{*} P<0.05$ using one-way ANOVA followed by Holm-Sidak testing for multiple comparisons. 
nerve growth during inflammation. Our immunohistochemical analysis revealed a CFA-induced upregulation of both the neuronal marker TUJ1 and the active phosphorylated form of ERK in the sciatic nerve (Figures 7A,B). Moreover, we observed that when conotoxin was injected intrathecally, post-CFA intraplantar injection, it inhibited the CFA mediated increase in TUJ1 expression and ERK phosphorylation (Figures 7A,B). Further quantification by western blot was consistent with our immunohistochemistry results (Figure 7C). Overall, our data strongly suggest that Cav2.2 is required for CFA-induced sensory nerve growth through indirect phosphorylation of the signaling molecule ERK. Along these lines, ERK phosphorylation likely promotes the expression of genes which control the sensory neurite overgrowth phenotype.

\section{DISCUSSION}

In the present study, we demonstrate that peripheral sensory nerve growth mediated via Cav2.2 activity drives inflammationinduced pain hypersensitivity. This conclusion is based on three lines of evidence. First, peripheral inflammation upregulates the expression of TUJ1, a post-mitotic neuritogenesis marker (von Bohlen, 2007) and Cav2.2 in the dorsal root ganglia. Second, pharmacological blockade of Cav2.2 channels attenuates inflammatory pain behavior in mice by preventing peripheral afferent growth. Third, inhibition of Cav2.2 results in downregulation of ERK1/2-MAP kinase, one of the major nociceptive signaling pathways associated with chronic inflammatory pain (Ji et al., 2002). Although persistent inflammation has been shown to alter density and distribution of a variety of voltage gated calcium channels (VGCCs) in the DRG (Lu et al., 2010), it remained unclear if VGCCs played any role in nerve growth post inflammation. Data from this study demonstrates that selective blockade of Cav2.2 with $\omega$-conotoxin alleviates the inflammatory pain hypersensitivity by preventing afferent growth and ERK1/2 activation (Figure 8).

Recent studies have shown that injection of inflammatory compounds, such as carrageenan and CFA leads to upregulation of voltage gated sodium channels (Nav), TRP channels, acidsensing ion channels (ASIC), and VGCCs in DRG neurons (Sgambato et al., 1998; Ji et al., 2002; von Bohlen, 2007; Yu et al., 2008). In particular, increased expression of TRPV1, TRPA1, and TRPM8 in primary afferents has been shown to be directly responsible for thermal and mechanical hypersensitivity in persistent inflammation (Mishra and Hoon, 2010; Chen et al., 2011; Mishra et al., 2011). N-type calcium channels have also been shown to play a major role in mediating release of synaptic transmitters like glutamate, acetylcholine, gamma-aminobutyric acid and calcitonin gene-related peptide (CGRP) (Nakanishi et al., 2010; Lalisse et al., 2018). Immunohistochemical evidence has also demonstrated increased localization of calcium channel subtypes in the dorsal horn of the spinal cord, nerve terminals and interneurons following injury and inflammation (Vigers and Pfenninger, 1991; Westenbroek et al., 1998; Cizkova et al., 2002). In addition, increased overlap of TRPV $1+$ and TRPA $1+$ sensory
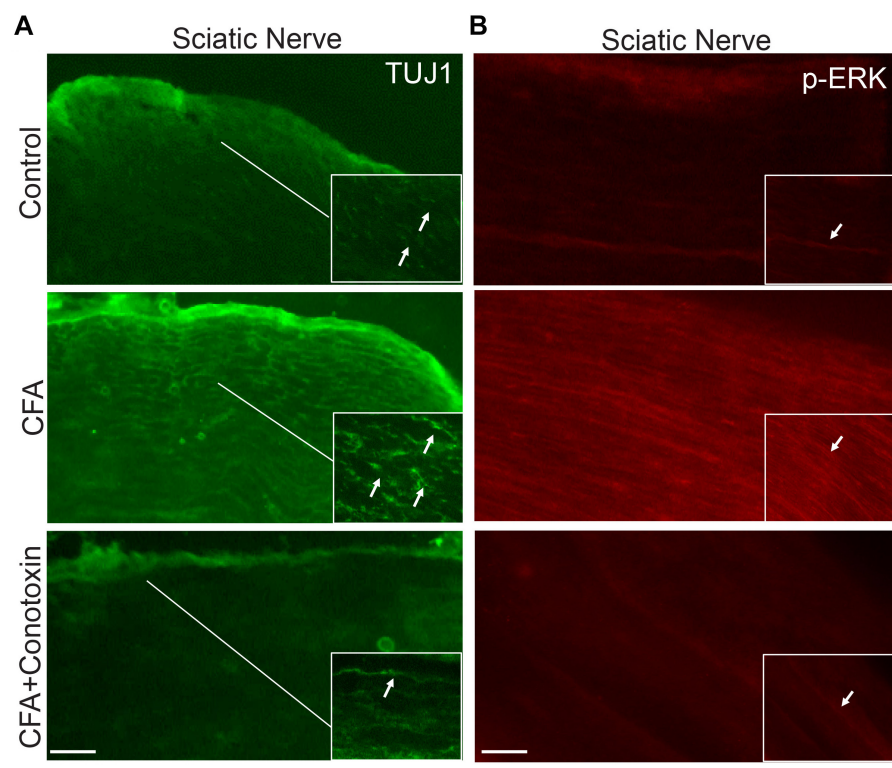

C
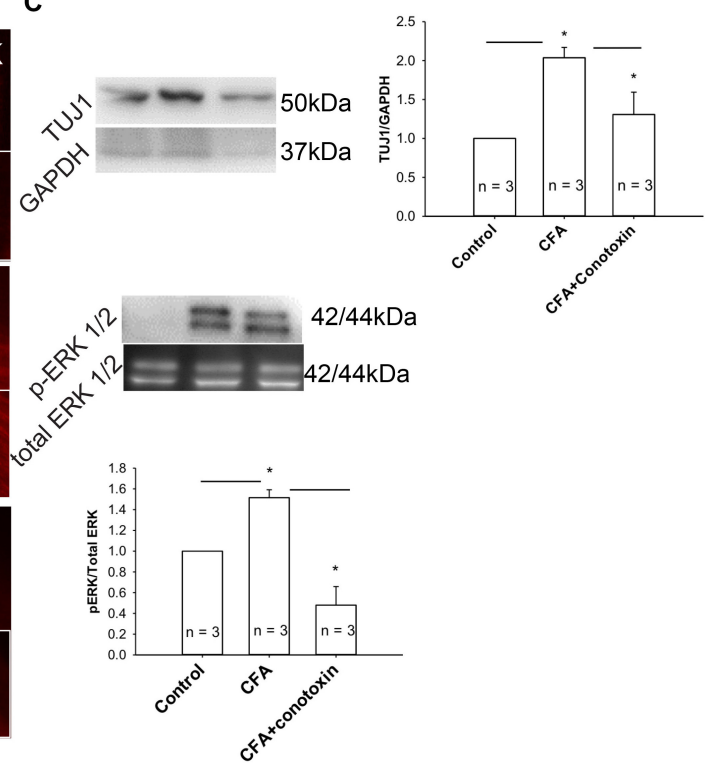

FIGURE 7 | Inflammation associated neurite growth is dependent on Cav2.2 activity and is driven via ERK activation. (A) Representative micrographs show sciatic nerve sections from control, CFA treated and CFA + $\omega$-conotoxin treated mice. A significant increase in TUJ1 expression can be seen post CFA treatment (white arrows in inset pointing at TUJ1 labeled afferents). $\omega$-conotoxin prevents CFA mediated increase in TUJ1 labeled afferents. $n=3$ mice per group. Scale bar $=30 \mu M$, inset scale bar $=40 \mu \mathrm{M}$. (B) Representative micrographs show phosphorylated-ERK1/2 immunolabeling. Conotoxin prevents CFA induced increase in phosphorylated ERK. (C) Immunoblots showing changes in TUJ1and pERK1/2 expression in sciatic nerve consistent with histochemical analysis. Conotoxin mitigates TUJ1 and p-ERK1/2 activity upon CFA induced inflammation, $n=3$ mice per group, ${ }^{*} P<0.01$ using one-way ANOVA followed by Holm-Sidak testing for multiple comparisons between groups. 


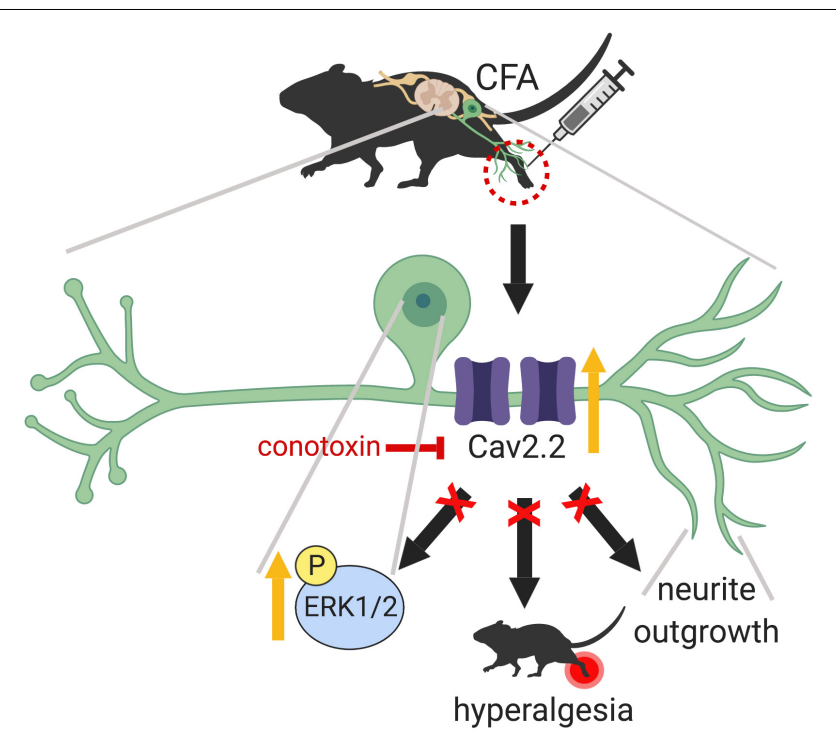

FIGURE 8 | Graphical abstract summarizing the main findings of this work which highlight a peripheral role for Cav2.2 during inflammation. CFA induced inflammation leads to increased Cav2.2 expression and ion channel activity which seem to drive peripheral afferent growth at the site of inflammation. One way that these changes could be manifesting is through activation of the ERK1/2-MAPK pathway further downstream. Together, these changes contribute to inflammation associated hyperalgesia. Intrathecal injection of $\omega$-conotoxin alleviates hyperalgesia by blocking Cav2.2 activity and associated afferent growth by downregulating ERK $1 / 2$. Schematic representation created with BioRender.com.

neurons in DRG with known pain neurotransmitters, such as substance $\mathrm{P}$ and CGRP post peripheral inflammation has been extensively reported and linked to thermal hyperalgesia (Nakanishi et al., 2010; Lalisse et al., 2018; Yu et al., 2008). Using RNA in situ hybridization, here we show a significant increase in Cav2.2 expression specifically in TRPV1+, TRPA1+, and TRPM8+ neuronal populations in ipsilateral DRGs $48 \mathrm{~h}$ post CFA-induced inflammation. Consistent with increased mRNA, total Cav2.2 protein expression was also upregulated in ipsilateral DRGs compared to contralateral DRGs. Together, these findings suggest that upregulation of Cav2.2 induced by peripheral inflammation might in turn cause increased TRP channel activity leading to further exacerbation of the condition. Interestingly, Cav2.2 was not upregulated in nonpeptidergic mechanosensitive nociceptors labeled by MrgprD (Dong et al., 2001; Lembo et al., 2002; Cavanaugh et al., 2009), or MrgprB4+ C-low threshold mechanoreceptors post CFA-injection. These cell-type specific gene expression changes observed with Cav2.2 during inflammation suggest that other VGCCs besides the N-type may contribute to mechanical allodynia observed during inflammation.

In regards to loss-of-function studies with Cav2.2, it has been reported that the genetic ablation of Cav2.2 or $\alpha 1 B$ (the pore forming subunit of Cav2.2) in mice, results in attenuation of chronic inflammatory pain induced by formalin and acetic acid (Hatakeyama et al., 2001; Kim et al., 2001). Although the overall anti-nociceptive effects of Cav2.2 ablation on thermal hyperalgesia are consistent, there are conflicting reports about the reduction in mechanical pain hypersensitivity mediated via Cav2.2 (Hopkins et al., 1995; Rigo et al., 2013). This seeming inconsistency could be due to the diversity in the expression pattern of calcium channels or unintended consequences of the specific genetic strategies used for ablation (Olivera et al., 1994). As an alternative to genetic ablation, conopeptides, which are natural and potent antagonists of N-type VGCCs have been used extensively in recent years (Olivera et al., 1987; Hopkins et al., 1995; Vanegas and Schaible, 2000; Lee, 2013). Relatedly, intrathecal administration of conopeptides is anti-nociceptive in acute inflammatory pain models (Olivera et al., 1987; Staats et al., 2004; Rigo et al., 2013). A recent study examined the efficacies of $\omega$-conotoxin MVIIA and Ph $\alpha 1 \beta$ in alleviating chemotherapy associated pain and reported positive outcomes (Rigo et al., 2013). Thus, in this study we likewise turned toward a pharmacological strategy with conotoxin to inhibit Cav2.2 activity during inflammatory states. We administered $\omega$-conotoxin GVIA (300 pmol) in the lumbar space by intrathecal injection after CFA induced inflammation at regular intervals for $72 \mathrm{~h}$. A significant reduction in thermal and cold pain sensitivities was observed, without effecting mechanical pain sensitivity. The sensory modality specific differences we observe by blocking Cav2.2 during inflammation, which differ from published reports, could be due to differences in the pharmacological properties of $\omega$-conotoxin GVIA and $\omega$-conotoxin MVIIA.

Sensory nerves involved in intra-plantar inflammation have cell bodies in the lumbar DRG. These DRGs process the signals received from their peripheral afferent projections and relay information to the CNS through the central projections in the dorsal horn of the spinal cord (Zhang and Dougherty, 2014). Persistent inflammation triggered by CFA injection or nerve injury generated via chronic constriction or nerve ligation, have been shown to amplify this signaling cascade (Lu and Gold, 2008; Lu et al., 2010). Intra-plantar injection of inflammatory soup in rats has been shown to increase the frequency of spontaneous neuronal discharge (Kasai and Mizumura, 2001; Ma et al., 2006). Multiple ion channels including $\mathrm{Na}^{+}, \mathrm{K}^{+}$have been shown to contribute to the lowering of action potential threshold and resting membrane potential in lumbar DRGs, which affects a large population of afferent neurons. In agreement with these findings, our voltage-clamp and current clamp analyses carried out post CFA treatment with and without $\omega$-conotoxin in lumbar (L4-L7) DRGs showed a significant increase in Cav2.2 current density and spontaneous action potentials, which were inhibited by $\omega$-conotoxin treatment. These findings implicate a role for Cav2.2 in increased excitability of neurons after inflammation.

An emerging literature has shown that a variety of factors contribute toward increased primary afferent growth at the onset on inflammation including cytokines, fibronectin, and nerve growth factor (Woolf et al., 1997; Miller et al., 2009; Tonge et al., 2012). Studies in Xenopus laevis and the pheochromocytoma (PC12) cell line suggest that calcium influx through VGCC causes growth cone extension, increases in connectivity, and neurite outgrowth (Solem et al., 1995; Spitzer, 2006; Sann et al., 2008). This prompted us to analyze if there were any changes in nerve growth following CFA-induced inflammation. 
DRGs, sciatic nerve, and hind paws harvested $24 \mathrm{~h}$ post intraplantar CFA injection revealed a significant increase in TUJ1, a neuronal marker which labels neurites. In addition, a robust increase in Cav2.2 surface expression in DRGs was also seen post inflammation. This led us to hypothesize that Cav2.2 might be driving neurite outgrowth. Remarkably, a significant reduction in the neuritogenesis marker TUJ1 was observed post $\omega$-conotoxin GVIA treatment across DRGs, sciatic nerve, and paws. This suggests a direct correlation between Cav2.2 activity and neuronal growth in peripheral inflammation.

Peripheral inflammation has been shown to activate immediate early genes like cFos (Xing et al., 1996; Sgambato et al., 1998) acutely and the ERK1/2-MAPK on a longer timescale (Ji et al., 2002). The functional role of ERK activation in neuronal physiology is very broad and includes neurite outgrowth. Elevated intracellular $\mathrm{Ca}^{2+}$ triggers various signaling pathways including protein kinases such as Calmodulin-dependent kinases (CaMK) and ERKs in neuroblastoma cell line. Additionally, ERK kinase inhibitor blocks BDNF-induced neurite outgrowth in neuroblastoma cell line SH-SY5Y (Encinas et al., 1999). Here, we showed a significant reduction in phosphorylated ERK (pERK1/2) upon $\omega$-conotoxin treatment in primary afferents. Therefore, in addition to inhibiting neuritogenesis during inflammation, blockade of Cav2.2 might be downregulating the ERK1/2-MAPK signaling cascade and alleviating inflammatory pain hypersensitivity. In addition, a recent HA-tagged Cav2.2 knock-mouse was generated and it was shown that Cav2.2 is expressed in the dorsal horn of the spinal cord in close apposition to terminal endings of nociceptive neurons (NietoRostro et al., 2018). Therefore, it is possible that in addition the peripheral changes we have demonstrated with Cav2.2 in this work, spinal cord changes in Cav2.2 may also drive components of inflammation induced hyperalgesia. Taken together, this work adds a new paradigm to what changes may be driving inflammation induced thermal hyperalgesia and suggests that strategies aimed at inhibiting neurite growth may serve as potent analgesics to block some forms of pain.

\section{REFERENCES}

Altier, C., Dale, C. S., Kisilevsky, A. E., Chapman, K., Castiglioni, A. J., Matthews, E. A., et al. (2007). Differential role of N-type calcium channel splice isoforms in pain. J. Neurosci. 27, 6363-6373. doi: 10.1523/jneurosci.0307-07. 2007

Amaya, F., Wang, H., Costigan, M., Allchorne, A. J., Hatcher, J. P., Egerton, J., et al. (2006). The voltage-gated sodium channel $\mathrm{Na}(\mathrm{v}) 1.9$ is an effector of peripheral inflammatory pain hypersensitivity. J. Neurosci. 26, 12852-12860. doi: 10.1523/jneurosci.4015-06.2006

Berta, T., Qadri, Y., Tan, P. H., and Ji, R. R. (2017). Targeting dorsal root ganglia and primary sensory neurons for the treatment of chronic pain. Expert Opin. Ther. Targets 21, 695-703. doi: 10.1080/14728222.2017.1328057

Brenner, D. S., Golden, J. P., and Gereau, R. W. IV (2012). A novel behavioral assay for measuring cold sensation in mice. PLoS One 7:e39765. doi: 10.1371/journal. pone.0039765

Cavanaugh, D. J., Lee, H., Lo, L., Shields, S. D., Zylka, M. J., Basbaum, A. I., et al. (2009). Distinct subsets of unmyelinated primary sensory fibers mediate behavioral responses to noxious thermal and mechanical stimuli. Proc. Natl. Acad. Sci. U.S.A. 106, 9075-9080. doi: 10.1073/pnas.0901507106

\section{DATA AVAILABILITY}

All datasets generated for this study are included in the manuscript and/or the Supplementary Files.

\section{ETHICS STATEMENT}

The animal study was reviewed and approved by North Carolina State University and the University of Pennsylvania Institutional Animal Care and Use Committee.

\section{AUTHOR CONTRIBUTIONS}

SP and LM designed and carried out the experiments. IA-S and SM designed the experiments. All authors contributed to the writing of the manuscript.

\section{FUNDING}

Major part of this work was supported by the NCSU startup funds to SM. This work was also supported by startup funds from the University of Pennsylvania to IA-S and NIDCR R00 grant (DE026807).

\section{SUPPLEMENTARY MATERIAL}

The Supplementary Material for this article can be found online at: https://www.frontiersin.org/articles/10.3389/fnins. 2019.01009/full\#supplementary-material

FIGURE S1 | Co-expression of Cav2.2 and the gentle touch sensor MrgprB4: Overlap of Cav2.2 and MrgprB4 with and without CFA injury. Contralateral DRGs (A-C) and Ipsilateral DRGs (D-F) show no significant change Cav2.2-MrgprB4 mRNA co-expression.

Chen, W. H., Hsieh, C. L., Huang, C. P., Lin, T. J., Tzen, J. T., Ho, T. Y., et al. (2011). Acid-sensing ion channel 3 mediates peripheral anti-hyperalgesia effects of acupuncture in mice inflammatory pain. J. Biomed. Sci. 18:82. doi: 10.1186/ 1423-0127-18-82

Cizkova, D., Marsala, J., Lukacova, N., Marsala, M., Jergova, S., Orendacova, J., et al. (2002). Localization of N-type Ca2+ channels in the rat spinal cord following chronic constrictive nerve injury. Exp. Brain Res. 147, 456-463. doi: 10.1007/s00221-002-1217-3

Dong, X., Han, S., Zylka, M. J., Simon, M. I., and Anderson, D. J. (2001). A diverse family of GPCRs expressed in specific subsets of nociceptive sensory neurons. Cell 106, 619-632. doi: 10.1016/s0092-8674(01)00483-4

Encinas, M., Iglesias, M., Llecha, N., and Comella, J. X. (1999). Extracellularregulated kinases and phosphatidylinositol 3-kinase are involved in brainderived neurotrophic factor-mediated survival and neuritogenesis of the neuroblastoma cell line SH-SY5Y. J. Neurochem. 73, 1409-1421. doi: 10.1046/j. 1471-4159.1999.0731409.x

Fehrenbacher, J. C., Vasko, M. R., and Duarte, D. B. (2012). Models of inflammation: carrageenan- or complete Freund's Adjuvant (CFA)-induced edema and hypersensitivity in the rat. Curr. Protoc. Pharmacol. 56, 5.4.1-5.4.4. doi: 10.1002/0471141755.ph0504s56 
Gold, M. S., and Flake, N. M. (2005). Inflammation-mediated hyperexcitability of sensory neurons. Neurosignals 14, 147-157. doi: 10.1159/000087653

Gu, X., and Spitzer, N. C. (1995). Distinct aspects of neuronal differentiation encoded by frequency of spontaneous Ca2+ transients. Nature 375, 784-787. doi: $10.1038 / 375784 \mathrm{a} 0$

Hatakeyama, S., Wakamori, M., Ino, M., Miyamoto, N., Takahashi, E., Yoshinaga, T., et al. (2001). Differential nociceptive responses in mice lacking the alpha(1B) subunit of $\mathrm{N}$-type $\mathrm{Ca}(2+)$ channels. Neuroreport 12, 2423-2427. doi: 10.1097/ 00001756-200108080-00027

Hillman, D., Chen, S., Aung, T. T., Cherksey, B., Sugimori, M., and Llinas, R. R. (1991). Localization of P-type calcium channels in the central nervous system. Proc. Natl. Acad. Sci. U.S.A. 88, 7076-7080. doi: 10.1073/pnas.88.16.7076

Hopkins, C., Grilley, M., Miller, C., Shon, K. J., Cruz, L. J., Gray, W. R., et al. (1995). A new family of Conus peptides targeted to the nicotinic acetylcholine receptor. J. Biol. Chem. 270, 22361-22367.

Ji, R. R., Befort, K., Brenner, G. J., and Woolf, C. J. (2002). ERK MAP kinase activation in superficial spinal cord neurons induces prodynorphin and NK-1 upregulation and contributes to persistent inflammatory pain hypersensitivity. J. Neurosci. 22, 478-485. doi: 10.1523/jneurosci.22-02-00478.2002

Kasai, M., and Mizumura, K. (2001). Increase in spontaneous action potentials and sensitivity in response to norepinephrine in dorsal root ganglion neurons of adjuvant inflamed rats. Neurosci. Res. 39, 109-113. doi: 10.1016/s01680102(00)00201-7

Kim, C., Jun, K., Lee, T., Kim, S. S., McEnery, M. W., Chin, H., et al. (2001). Altered nociceptive response in mice deficient in the alpha(1B) subunit of the voltage-dependent calcium channel. Mol. Cell. Neurosci. 18, 235-245. doi: 10.1006/mcne.2001.1013

Lalisse, S., Hua, J., Lenoir, M., Linck, N., Rassendren, F., and Ulmann, L. (2018). Sensory neuronal P2RX4 receptors controls BDNF signaling in inflammatory pain. Sci. Rep. 8:964. doi: 10.1038/s41598-018-19301-5

Lankford, K. L., Waxman, S. G., and Kocsis, J. D. (1998). Mechanisms of enhancement of neurite regeneration in vitro following a conditioning sciatic nerve lesion. J. Comp. Neurol. 391, 11-29. doi: 10.1002/(sici)10969861(19980202)391:1<11::aid-cne2>3.0.co;2-u

Lee, S. (2013). Pharmacological inhibition of voltage-gated $\mathrm{Ca}(2+)$ channels for chronic pain relief. Curr. Neuropharmacol. 11, 606-620. doi: 10.2174/ 1570159X11311060005

Lembo, P. M., Grazzini, E., Groblewski, T., O’Donnell, D., Roy, M. O., Zhang, J., et al. (2002). Proenkephalin A gene products activate a new family of sensory neuron-specific GPCRs. Nat. Neurosci. 5, 201-209. doi: 10.1038/ nn 815

Lu, S. G., and Gold, M. S. (2008). Inflammation-induced increase in evoked calcium transients in subpopulations of rat dorsal root ganglion neurons. Neuroscience 153, 279-288. doi: 10.1016/j.neuroscience.2008.02.006

Lu, S. G., Zhang, X. L., Luo, Z. D., and Gold, M. S. (2010). Persistent inflammation alters the density and distribution of voltage-activated calcium channels in subpopulations of rat cutaneous DRG neurons. Pain 151, 633-643. doi: 10.1016/ j.pain.2010.08.030

Ma, C., Greenquist, K. W., and Lamotte, R. H. (2006). Inflammatory mediators enhance the excitability of chronically compressed dorsal root ganglion neurons. J. Neurophysiol. 95, 2098-2107. doi: 10.1152/jn.00748.2005

Miller, R. J., Jung, H., Bhangoo, S. K., and White, F. A. (2009). Cytokine and chemokine regulation of sensory neuron function. Handb. Exp. Pharmacol. 194, 417-449. doi: 10.1007/978-3-540-79090-7_12

Mishra, S. K., and Hoon, M. A. (2010). Ablation of TrpV1 neurons reveals their selective role in thermal pain sensation. Mol. Cell. Neurosci. 43, 157-163. doi: 10.1016/j.mcn.2009.10.006

Mishra, S. K., Tisel, S. M., Orestes, P., Bhangoo, S. K., and Hoon, M. A. (2011). TRPV1-lineage neurons are required for thermal sensation. EMBO J. 30, 582593. doi: $10.1038 /$ emboj.2010.325

Nakanishi, M., Hata, K., Nagayama, T., Sakurai, T., Nishisho, T., Wakabayashi, H., et al. (2010). Acid activation of Trpvl leads to an up-regulation of calcitonin gene-related peptide expression in dorsal root ganglion neurons via the CaMKCREB cascade: a potential mechanism of inflammatory pain. Mol. Biol. Cell 21, 2568-2577. doi: 10.1091/mbc.E10-01-0049

Nieto-Rostro, M., Ramgoolam, K., Pratt, W. S., Kulik, A., and Dolphin, A. C. (2018). Ablation of alpha2delta-1 inhibits cell-surface trafficking of endogenous
$\mathrm{N}$-type calcium channels in the pain pathway in vivo. Proc. Natl. Acad. Sci. U.S.A. 115, E12043-E12052. doi: 10.1073/pnas.1811212115

Olivera, B. M., Cruz, L. J., de Santos, V., LeCheminant, G. W., Griffin, D., Zeikus, R., et al. (1987). Neuronal calcium channel antagonists. Discrimination between calcium channel subtypes using omega-conotoxin from Conus magus venom. Biochemistry 26, 2086-2090. doi: 10.1021/bi00382a004

Olivera, B. M., Miljanich, G. P., Ramachandran, J., and Adams, M. E. (1994). Calcium channel diversity and neurotransmitter release: the omega-conotoxins and omega-agatoxins. Annu. Rev. Biochem. 63, 823-867. doi: 10.1146/annurev. biochem.63.1.823

Rigo, F. K., Dalmolin, G. D., Trevisan, G., Tonello, R., Silva, M. A., Rossato, M. F., et al. (2013). Effect of omega-conotoxin MVIIA and Phalpha1beta on paclitaxelinduced acute and chronic pain. Pharmacol. Biochem. Behav. 11, 16-22. doi: 10.1016/j.pbb.2013.10.014

Sann, S. B., Xu, L., Nishimune, H., Sanes, J. R., and Spitzer, N. C. (2008). Neurite outgrowth and in vivo sensory innervation mediated by a $\mathrm{Ca}(\mathrm{V}) 2.2$-laminin beta 2 stop signal. J. Neurosci. 28, 2366-2374. doi: 10.1523/JNEUROSCI.382807.2008

Schaible, H. G., Nebe, J., Neugebauer, V., Ebersberger, A., and Vanegas, H. (2000). The role of high-threshold calcium channels in spinal neuron hyperexcitability induced by knee inflammation. Prog. Brain Res. 129, 173-190. doi: 10.1016/ s0079-6123(00)29013-4

Sgambato, V., Pages, C., Rogard, M., Besson, M. J., and Caboche, J. (1998). Extracellular signal-regulated kinase (ERK) controls immediate early gene induction on corticostriatal stimulation. J. Neurosci. 18, 8814-8825. doi: 10. 1523/jneurosci.18-21-08814.1998

Snutch, T. P. (2005). Targeting chronic and neuropathic pain: the N-type calcium channel comes of age. NeuroRX 2, 662-670. doi: 10.1602/neurorx.2.4.662

Solem, M., McMahon, T., and Messing, R. O. (1995). Depolarization-induced neurite outgrowth in PC12 cells requires permissive, low level NGF receptor stimulation and activation of calcium/calmodulin-dependent protein kinase. J. Neurosci. 15, 5966-5975. doi: 10.1523/jneurosci.15-09-05966.1995

Spitzer, N. C. (2006). Electrical activity in early neuronal development. Nature 444, 707-712. doi: 10.1038/nature05300

Staats, P. S., Yearwood, T., Charapata, S. G., Presley, R. W., Wallace, M. S., ByasSmith, M., et al. (2004). Intrathecal ziconotide in the treatment of refractory pain in patients with cancer or AIDS: a randomized controlled trial. JAMA 291, 63-70.

Tonge, D. A., de Burgh, H. T., Docherty, R., Humphries, M. J., Craig, S. E., and Pizzey, J. (2012). Fibronectin supports neurite outgrowth and axonal regeneration of adult brain neurons in vitro. Brain Res. 1453, 8-16. doi: 10.1016/ j.brainres.2012.03.024

Vanegas, H., and Schaible, H. (2000). Effects of antagonists to high-threshold calcium channels upon spinal mechanisms of pain, hyperalgesia and allodynia. Pain 85, 9-18. doi: 10.1016/s0304-3959(99)00241-9

Vigers, A. J., and Pfenninger, K. H. (1991). N-type and L-type calcium channels are present in nerve growth cones. Numbers increase on synaptogenesis. Brain Res. Dev. Brain Res. 60, 197-203. doi: 10.1016/0165-3806(91)90048-n

von Bohlen, U. H. O. (2007). Immunohistological markers for staging neurogenesis in adult hippocampus. Cell Tissue Res. 329, 409-420. doi: 10.1007/s00441-007$0432-4$

Weiss, N. (2008). The N-type voltage-gated calcium channel: when a neuron reads a map. J. Neurosci. 28, 5621-5622. doi: 10.1523/jneurosci.1538-08.2008

Westenbroek, R. E., Hell, J. W., Warner, C., Dubel, S. J., Snutch, T. P., and Catterall, W. A. (1992). Biochemical properties and subcellular distribution of an N-type calcium channel alpha 1 subunit. Neuron 9, 1099-1115. doi: 10.1016/08966273(92)90069-p

Westenbroek, R. E., Hoskins, L., and Catterall, W. A. (1998). Localization of $\mathrm{Ca} 2+$ channel subtypes on rat spinal motor neurons, interneurons, and nerve terminals. J. Neurosci. 18, 6319-6330. doi: 10.1523/jneurosci.18-16-06319.1998

Woolf, C. J., Allchorne, A., Safieh-Garabedian, B., and Poole, S. (1997). Cytokines, nerve growth factor and inflammatory hyperalgesia: the contribution of tumour necrosis factor alpha. Br. J. Pharmacol. 121, 417-424. doi: 10.1038/sj.bjp. 0701148

Xing, J., Ginty, D. D., and Greenberg, M. E. (1996). Coupling of the RAS-MAPK pathway to gene activation by RSK2, a growth factor-regulated CREB kinase. Science 273, 959-963. doi: 10.1126/science.273.5277.959 
Xu, G. Y., and Huang, L. Y. (2002). Peripheral inflammation sensitizes P2X receptor-mediated responses in rat dorsal root ganglion neurons. J. Neurosci. 22, 93-102. doi: 10.1523/jneurosci.22-01-00093.2002

Yu, L., Yang, F., Luo, H., Liu, F. Y., Han, J. S., Xing, G. G., et al. (2008). The role of TRPV1 in different subtypes of dorsal root ganglion neurons in rat chronic inflammatory nociception induced by complete Freund's adjuvant. Mol Pain 4:61. doi: 10.1186/1744-8069-4-61

Zamburlin, P., Ruffinatti, F. A., Gilardino, A., Farcito, S., Parrini, M., and Lovisolo, D. (2013). Calcium signals and FGF-2 induced neurite growth in cultured parasympathetic neurons: spatial localization and mechanisms of activation. Pflugers Arch. 465, 1355-1370. doi: 10.1007/s00424-0131257-5

Zamponi, G. W., Striessnig, J., Koschak, A., and Dolphin, A. C. (2015). the physiology, pathology, and pharmacology of voltage-gated calcium channels and their future therapeutic potential. Pharmacol. Rev. 67, 821-870. doi: 10. $1124 /$ pr.114.009654
Zhang, H., and Dougherty, P. M. (2014). Enhanced excitability of primary sensory neurons and altered gene expression of neuronal ion channels in dorsal root ganglion in paclitaxel-induced peripheral neuropathy. Anesthesiology 120, 1463-1475. doi: 10.1097/aln.0000000000000176

Conflict of Interest Statement: The authors declare that the research was conducted in the absence of any commercial or financial relationships that could be construed as a potential conflict of interest.

Copyright (C) 2019 Pitake, Middleton, Abdus-Saboor and Mishra. This is an openaccess article distributed under the terms of the Creative Commons Attribution License (CC BY). The use, distribution or reproduction in other forums is permitted, provided the original author(s) and the copyright owner(s) are credited and that the original publication in this journal is cited, in accordance with accepted academic practice. No use, distribution or reproduction is permitted which does not comply with these terms. 